\title{
Parametric analysis and optimization of convective fin with variable thermal conductivity using semi-analytical solution
}

\author{
Vadivelu M. Arumugam*, Ramesh K. Chidambaram \\ Automotive Research Center, Department of Automotive Engineering, School of Mechanical Engineering, VIT University, \\ Vellore 632014, India
}

Corresponding Author Email: vadivelu.m.arumugam@gmail.com

https://doi.org/10.18280/ijht.360233

Received: 6 October 2017

Accepted: 27 March 2018

\section{Keywords:}

heat transfer performance, temperature dependent thermal conductivity, straight fins, ADM, optimization and parametric analysis

\begin{abstract}
Due to the temperature dependent properties, evaluation of heat transfer performance parameters of a polymer composite material through experimentation is difficult as it needs sophisticated measurement techniques. In this article, to meet the current requirements, a simple semi-analytical method is proposed to investigate the performance of convective straight fins with temperature dependent thermal conductivity. The Adomian Decomposition Method (ADM) was adopted to solve the non-linear energy equation and Newton-Raphson method was used for optimization of the fin problem. After the analysis, the effect of convective-geometric fin parameter and thermal conductivity parameter is introduced in this problem to interpret the physical significance of such parameters. A parametric analysis was carried out to depict the dependency of heat transfer phenomena on various parameters. The informative plot on the gradient field of the fin efficiency negotiates the direction of maximum performance.
\end{abstract}

\section{INTRODUCTION}

The requirement of the present day heat exchanging equipment is a high surface area to volume ratio, light weight, compactness, manufacturability and low manufacturingmaintenance cost. Particularly, in case of atmospheric air dependent cross flow heat exchangers like plate fin heat exchangers and tube finned heat exchangers need high surface area to volume ratio of the fin structures to improve air side heat transfer rate [1-2]. In the past decade, a lot of research work was carried out to overcome air side resistance during the heat transfer process by varying the geometry of the fin structures. To improve the heat transfer rate, the adoption of the newly developed fin structures is mandatory.

The manufacturing, material and maintenance (MMM) flexibility and cost plays a major role during the production of new fin structures. With the help of the high conductivity polymer composite materials, the MMM flexibility can be maintained under required limits compared with conventional metals due to the good mechanical-economical values of the polymers.

Polymer composite materials are widely used in various engineering applications to enhance the mechanical properties, life of the product and also to reduce the global cost consumption in the product development stream. The thermal properties of the polymer composite materials are very poor whencompared with all other metals, metalloids [3]. Polymer composite materials exhibit low thermal properties due to poor phonon energy transfer in the sub-atomic level and the heat transfer capacity depends on the qualitative and quantitative nature of the reinforcing materials $[4,6]$. For developing a high thermal conductivity polymeric composite material, the investigation on heat transfer behavior of such material is a necessary one to execute inverse heat transfer problem to design such a composite. Experimental investigation on heat transfer behavior of composite material is complex method because it requires sophisticated measurement techniques due to the anisotropic behavior of the material. Thermal conductivity of the composite material is a dynamic property which varies with respect to temperature due to the anisotropic nature [5-6].

In fact, a considerable amount of research has been conducted considering temperature dependent thermal conductivity parameter which is associated with metal fins operating in practical situations. But the investigations on the thermal performance of variable thermal conductivity material based fins showed poor information regarding design parameters of such a fin system. The governing equation for fin model with variable thermal conductivity is a non-linear differential equation and in most of the cases, numerical schemes are adopted to solve the problems. Initiatively, Hung and App [7] investigated on variable thermal conductivity system with internal heat generation. Unal [8] announced a solution based on series of studies on an extended surface of non-uniform heat transfer coefficient with limited number of cases.Later, Meyers [9] analyzed on the steady periodic heat conduction in a semi-infinite medium and extended it by the method of complex combination. Muzzio [10] explored approximate solutions for the temperature dependent thermal conductivity fin problem based on the Galerkin method, which involves selection of suitable basis function. The parametric performance analysis and optimization of the fin design parameter are being major voids in the past research progress. The direction for achieving maximum performance per minimum design constraints is also not interpreted in the literatures.

In most cases, these kinds of problem do not declare analytical solution, so these nonlinear equations can be solved 
by special techniques. The integral transform methods, Laplace transforms and Fourier transforms were successfully adopted for these kinds of problem [11-15] with linear equations. But for nonlinear problems, these methods result in more complex and difficult solutions. Recently, the Adomian Decomposition Method (ADM) [16-17] has emerged as a semi-analytic scheme to solve a non-linear problems whose mathematical models possesses algebraic [18], differential and partial differential equations $[19,20]$. The main advantage of the decomposition method is that it solves both deterministic and stochastic non-linear problems without any linearization scheme [21].

In this communication, analytical solution of parametric performance analysis of temperature dependent thermal conductivity straight fins with convective environment has been studied by Adomian decomposition method. For this purpose, after portrayal of the problem statement-assumptions and brief introduction of Adomian Decomposition Method (ADM), ADM was adopted to produce approximate solution for the given problem. For an isotropic fin case study, procurement of result and comparing it with exact solution reveals the compatibility, effectiveness and adherence of ADM for such a kind of problems. The efficiency and nondimensional heat transfer rate of the fin is derived and the direction for maximum performance with minimum design requirement was identified using optimization technique.

\section{MATHEMATICAL MODEL AND ASSUMPTIONS}

One dimensional composite extended surface (fin) with a temperature dependent thermal conductivity $\mathrm{k}(\mathrm{T})$, perimeter $\mathrm{P}$, length $\mathrm{L}$, thickness $\mathrm{t}$ and fixed cross-sectional area $\mathrm{A}_{\mathrm{c}}$ was considered. The uniform bottom surface of the straight fin is attached with an isotropic metal surface of uniform temperature $T_{b}$ and the tip of fin is insulated which roots to adiabatic nature. Figure 1 depicts an illustration of the fin geometry, where the axial distance $\mathrm{x}$ is measured from the tip end. The fin is exposed to the convective environment of a temperature $T_{a}$ and a convective heat transfer coefficient of $h$. The following assumptions were considered to solve the present composite fin problem:

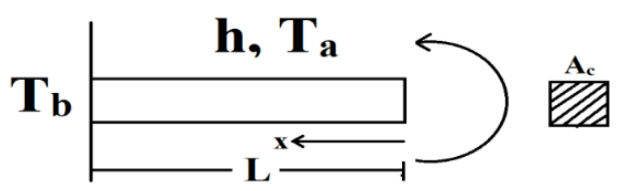

Figure 1. Illustration of a rectangular straight fin

1. Composite fin material is heterogeneous in nature and it exhibits variable thermal conductivity.

2. The physical properties of the surrounding fluid medium are invariable and fixed.

3. Temperature variation inside the fin is one-dimensional (preferably longitudinal direction).

4. Thermal conductivity $\mathrm{k}$ is the dependent variable and a function of a single variable temperature $\mathrm{T}$ at each point of the fin.

5. There is no bond resistance at the fin base and there are no heat producing sources in the fin itself.

6. Heat transferred through the outer most edge of the fin is negligible compared with its sides (Adiabatic tip condition).
In this study, total heat transfer from the composite fin is calculated as the summation of convection due to motion of the fluid flowing around the fin and conduction in the fin. By employing an energy conservation law, mathematically it represents

$\frac{\partial}{\partial \mathrm{x}}\left(\mathrm{k} \frac{\partial \mathrm{T}}{\partial \mathrm{x}}\right)-\frac{\mathrm{hP}}{\mathrm{A}_{\mathrm{c}}}\left(\mathrm{T}-\mathrm{T}_{\mathrm{a}}\right)=0$

The temperature dependent thermal conductivity of the composite material is defined by

$\mathrm{k}(\mathrm{T})=\mathrm{k}_{\mathrm{a}}\left[1+\Omega\left(\mathrm{T}-\mathrm{T}_{\mathrm{a}}\right)\right]$

and the boundary conditions can be detailed as

$\left.\frac{\partial \mathrm{T}}{\partial \mathrm{x}}\right|_{\mathrm{x}=0}=0 \quad$ and $\left.\quad \mathrm{T}\right|_{\mathrm{x}=\mathrm{L}}=\mathrm{T}_{\mathrm{b}}$

by employing equations (2), (3) and the following dimensionless variables, parameters and constants,

$\theta=\frac{\mathrm{T}-\mathrm{T}_{\mathrm{a}}}{\mathrm{T}_{\mathrm{b}}-\mathrm{T}_{\mathrm{a}}}, \gamma=\frac{\mathrm{x}}{\mathrm{L}}, \zeta=\frac{\mathrm{k}_{\mathrm{b}}-\mathrm{k}_{\mathrm{a}}}{\mathrm{k}_{\mathrm{a}}}=\Omega\left(\mathrm{T}_{\mathrm{b}}-\mathrm{T}_{\mathrm{a}}\right), \mathrm{N}^{2}=\frac{\mathrm{hPL}^{2}}{\mathrm{k}_{\mathrm{a}} \mathrm{A}_{\mathrm{c}}}$

$\frac{d^{2} \theta}{d \gamma^{2}}+\zeta \theta\left(\frac{d^{2} \theta}{d \gamma^{2}}\right)+\zeta\left(\frac{d \theta}{d \gamma}\right)^{2}-N^{2} \theta=0$

subjected to the boundary conditions of

$\left.\frac{\mathrm{d} \theta}{\mathrm{d} \gamma}\right|_{\gamma=0}=0,\left.\quad \theta\right|_{\gamma=1}=1$

We consider the range of dimensionless composite fin parameters as

$0 \leq \mathrm{N} \leq 1.5$ and $0 \leq \gamma \leq 1$

\section{DECOMPOSITION METHOD FOR NONLINEAR EQUATION}

The general nonlinear equations cannot be solved with the help of usual analytical techniques. Currently, application of decomposition method is popular for solving the nonlinear sorts of equations. The Adomian decomposition method was introduced and elaborated in late 1980 s to solve boundary based physical problems. The general non-linear equation represented by the Adomian decomposition method is in the form of

$(\mathrm{L}+\mathrm{R}) \sigma+\mathrm{n} \sigma-\mathrm{g}=0$.

The linear and non-linear terms of an algorithm were decomposed into $(\mathrm{L}+\mathrm{R}) \sigma$ and $\mathrm{n} \sigma$ operators respectively [21]. The higher order term of the linear operators is considered as $\mathrm{L}$ term to avoid complex integration problem involving difficult green's function, and the remaining is termed as remainder $\mathrm{R}$. The define integration from 0 to $\sigma$ is used to develop the inverse operator $\mathrm{L}^{-1}$ of the linear operator $\mathrm{L}$, i.e.

$\left[\mathrm{L}^{-1} \mathrm{f}\right](\sigma)=\int_{0}^{\sigma} \mathrm{f}\left(\sigma^{1}\right) \mathrm{d} \sigma$

If $\mathrm{L}$ is an $\mathrm{n}^{\text {th }}$ order differential operator, then $\mathrm{L}^{-1}$ will be an 
n-fold indefinite integral, i.e.

$\mathrm{L}^{-1}(\mathrm{~L} \sigma)=\mathrm{L}^{-1}\left(\frac{\mathrm{d}^{\mathrm{n}} \sigma}{\mathrm{d} \lambda^{\mathrm{n}}}\right)=$

$\int_{0}^{\sigma} \int_{0}^{\sigma} \int_{0}^{\sigma} \ldots \ldots \ldots \ldots \int_{0}^{\sigma}\left(\frac{\mathrm{d}^{\mathrm{n}} \sigma}{\mathrm{d} \lambda^{\mathrm{n}}}\right) \mathrm{d} \lambda \mathrm{d} \lambda \mathrm{d} \lambda \mathrm{d} \lambda \ldots \ldots \mathrm{d} \lambda$.

for a two-fold indefinite integration, it will be written as

$L^{-1}(L \sigma)=L^{-1}\left(\frac{d^{2} \sigma}{d \lambda^{2}}\right)=\int_{0}^{\sigma} \int_{0}^{\sigma}\left[\left(\frac{d^{2} \sigma}{d \lambda^{2}}\right) d \lambda\right] d \lambda=\sigma-\sigma(0)-$ $\lambda \frac{\partial \sigma}{\partial \lambda}(0)$.

based on the equation (8), the required formulation is

$\mathrm{L}^{-1}(\mathrm{~L} \sigma)=\mathrm{L}^{-1} \mathrm{~g}-\mathrm{L}^{-1} \mathrm{R} \sigma-\mathrm{L}^{-1} \mathrm{~N} \sigma$

and reduces to

$\sigma=\sigma(0)+\lambda \frac{\partial \sigma}{\partial \lambda}(0)+\mathrm{L}^{-1} \mathrm{~g}-\mathrm{L}^{-1} \mathrm{R} \sigma-\mathrm{L}^{-1} \mathrm{~N} \sigma$

For boundary value problems, above equation is further reduced into

$\sigma=\sigma_{0}-\mathrm{L}^{-1} \mathrm{R} \sigma-\mathrm{L}^{-1} \mathrm{~N} \sigma$

where $\sigma_{0}=\sigma(0)+\lambda \frac{\partial \sigma}{\partial \lambda}(0)+L^{-1} g$.

Based on decomposition method, a series solution of the equation (14) is written as

$\sum_{\mathrm{m}=0}^{\infty} \sigma_{\mathrm{m}}=\sigma_{0}-\mathrm{L}^{-1} \mathrm{R} \sum_{\mathrm{m}=0}^{\infty} \sigma_{\mathrm{m}}-\mathrm{L}^{-1} \sum_{\mathrm{m}=0}^{\infty} \mathrm{A}_{\mathrm{m}}$,

where $\mathrm{A}_{\mathrm{m}}$ are Adomian's polynomials of $\sigma_{0}, \sigma_{1}, \sigma_{2}, \ldots \ldots \ldots, \sigma_{m}$.

\section{PARAMETRIC TEMPERATURE DISTRIBUTION}

From decomposition analysis, the non-linear differential equation (5) of given fin problem can be detailed as follows:

$L_{\gamma} \theta=N^{2} \theta-\zeta \theta\left(\frac{d^{2} \theta}{d \gamma^{2}}\right)-\zeta\left(\frac{d \theta}{d \gamma}\right)^{2}$

Using equations (12) and (16), the above problem can be written as

$\sum_{\mathrm{m}=0}^{\infty} \theta_{\mathrm{m}}=\theta_{0}+\mathrm{L}^{-1} \mathrm{~N}^{2} \sum_{\mathrm{m}=0}^{\infty} \theta_{\mathrm{m}}-\mathrm{L}^{-1} \zeta \sum_{\mathrm{m}=0}^{\infty} \mathrm{A}_{\mathrm{m}}-$

$\mathrm{L}^{-1} \zeta \sum_{\mathrm{m}=0}^{\infty} \mathrm{B}_{\mathrm{m}}$

reduced into

$\sum_{\mathrm{m}=0}^{\infty} \theta_{\mathrm{m}+1}=\mathrm{N}^{2} \mathrm{~L}^{-1} \sum_{\mathrm{m}=0}^{\infty} \theta_{\mathrm{m}}-\zeta \mathrm{L}^{-1} \sum_{\mathrm{m}=0}^{\infty} \mathrm{A}_{\mathrm{m}}-$

$\zeta \mathrm{L}^{-1} \sum_{\mathrm{m}=0}^{\infty} \mathrm{B}_{\mathrm{m}}$,

where $\theta_{0}=\theta(0)+\gamma \frac{\partial \theta}{\partial \gamma}(0)$, the non-linear terms in the equation are decomposed into

$\theta\left(\frac{d^{2} \theta}{d \gamma^{2}}\right)=\sum_{m=0}^{\infty} A_{m}$,

$\left(\frac{\mathrm{d} \theta}{\mathrm{d} \gamma}\right)^{2}=\sum_{\mathrm{m}=0}^{\infty} \mathrm{B}_{\mathrm{m}}$.
Temperature distribution of the given well-posed fin problem can be sentenced as (from Eq. 19)

$\theta(\gamma)=\sum_{\mathrm{i}=0}^{\mathrm{m}-1} \theta_{\mathrm{i}}=\theta_{0}+\theta_{1}+\theta_{2}+\theta_{3}+\cdots+\theta_{\mathrm{m}-1}, \quad$ for $\mathrm{m} \geq 0$

and the first term in the series is derived from equation (15), other are followed by recursive iteration

$\theta_{\mathrm{m}+1}=\mathrm{N}^{2} \mathrm{~L}^{-1} \theta_{\mathrm{m}}-\zeta \mathrm{L}^{-1} \mathrm{~A}_{\mathrm{m}}-\zeta \mathrm{L}^{-1} \mathrm{~B}_{\mathrm{m}}$.

The decomposed non-linear terms in the given problem are represented by using Adomian's polynomials as

$A_{0}=\theta_{0}\left(\frac{d^{2} \theta_{0}}{d \gamma^{2}}\right)$,

$A_{1}=\theta_{1}\left(\frac{d^{2} \theta_{0}}{d \gamma^{2}}\right)+\theta_{0}\left(\frac{d^{2} \theta_{1}}{d \gamma^{2}}\right)$,

$A_{2}=\theta_{2}\left(\frac{d^{2} \theta_{0}}{d \gamma^{2}}\right)+\theta_{1}\left(\frac{d^{2} \theta_{1}}{d \gamma^{2}}\right)+\theta_{0}\left(\frac{d^{2} \theta_{2}}{d \gamma^{2}}\right), \ldots \ldots \ldots .$.

similarly,

$\mathrm{B}_{0}=\left(\frac{\mathrm{d} \theta_{0}}{\mathrm{~d} \gamma}\right)^{2}$,

$B_{1}=2\left(\frac{d \theta_{0}}{d \gamma}\right)\left(\frac{d \theta_{1}}{d \gamma}\right)$

$B_{2}=2\left(\frac{d \theta_{0}}{d \gamma}\right)\left(\frac{d \theta_{2}}{d \gamma}\right)+\left(\frac{d \theta_{1}}{d \gamma}\right)^{2}$

Based on equation (21), temperature distribution in the variable thermal conductivity composite fin is written in decomposed form as

$\theta_{0}=\tau$,

$\theta_{1}=\frac{1}{2} \tau N^{2} \gamma^{2}$

$\theta_{2}=\frac{1}{24} \tau N^{4} \gamma^{4}-\frac{1}{2} \zeta \tau^{2} N^{2} \gamma^{2}$,

$\theta_{3}=\frac{1}{720} \tau N^{6} \gamma^{6}-\frac{5}{24} \zeta \tau^{2} N^{4} \gamma^{4}+\frac{1}{2} \zeta^{2} \tau^{3} N^{2} \gamma^{2}$,

$\theta_{4}=\frac{1}{40320} \tau N^{8} \gamma^{8}-\frac{1}{45} \zeta \tau^{2} N^{6} \gamma^{6}+\frac{1}{2} \zeta^{2} \tau^{3} N^{4} \gamma^{4}-$

$\frac{1}{2} \zeta^{3} \tau^{4} N^{2} \gamma^{2}$

From equation (20), by summing up all the decomposed elements, temperature distribution of the fin as a function of dimensionless parameters can be expressed as

$\theta(\gamma)=\tau+\frac{1}{2} \tau N^{2} \gamma^{2}+\frac{1}{24} \tau N^{4} \gamma^{4}-\frac{1}{2} \zeta \tau^{2} N^{2} \gamma^{2}+$

$\frac{1}{720} \tau N^{6} \gamma^{6}-\frac{5}{24} \zeta \tau^{2} N^{4} \gamma^{4}+\frac{1}{2} \zeta^{2} \tau^{3} N^{2} \gamma^{2}+\frac{1}{40320} \tau N^{8} \gamma^{8}-$

$\frac{1}{45} \zeta \tau^{2} N^{6} \gamma^{6}+\frac{1}{2} \zeta^{2} \tau^{3} N^{4} \gamma^{4}-\frac{1}{2} \zeta^{3} \tau^{4} N^{2} \gamma^{2}+\cdots$,

where

$\theta_{0}=\theta(0)+\gamma \frac{\partial \theta}{\partial \gamma}(0)=\tau$

The co-efficient $\tau$ is determined by solving non-linear algebraic equation (27) using Newton-Raphson method with the help of specific boundary conditions as described in equation (6).

The required non-linear algebraic equation is 
$\left(\frac{1}{2} \zeta^{4} N^{2}\right) \tau^{5}-\left(\frac{1}{2} \zeta^{3} N^{2}+\frac{5}{6} \zeta^{3} N^{4}\right) \tau^{4}+\left(\frac{1}{2} \zeta^{2} N^{2}+\frac{1}{2} \zeta^{2} N^{4}+\right.$ $\left.\frac{49}{360} \zeta^{2} N^{6}\right) \tau^{3}-\left(\frac{1}{2} \zeta N^{2}+\frac{5}{4} \zeta N^{4}+\frac{1}{45} \zeta N^{6}+\frac{37}{20160} \zeta N^{8}\right) \tau^{2}+$ $\left(1+\frac{1}{2} N^{2}+\frac{1}{24} N^{4}+\frac{1}{720} N^{6}+\frac{1}{40320} N^{8}+\frac{1}{3628800} N^{10}\right) \tau-$ $1=0$.

The above equation (25), (26) and (27) gives the approximate analytical solution of $\theta$ for assigned values of parameters, fin parameter $\mathrm{N}$, thermal conductivity parameter $\zeta$. With the help of all, therefore the temperature distribution of the fin in axial direction is expressed explicitly as a function of $\gamma(\mathrm{x})$ and the performance analysis of fin can be easily exhibited by the use of above mentioned equations.

The values of co-efficient $\tau$ expressed in terms of thermal conductivity parameter $\zeta$ and fin parameter $\mathrm{N}$ is calculated and plotted in figure 2 based on respective boundary conditions. The temperature distribution profile over the length of the fin depends on the characteristic value $\tau$ and the characteristic value increases at fin tip as the thermal conductivity parameter $\zeta$ of the fin increases. As expected, temperature at the tip of the fin is in reducing nature as the fin length increases. The above mentioned interpretations are shown clearly in figure 2 .

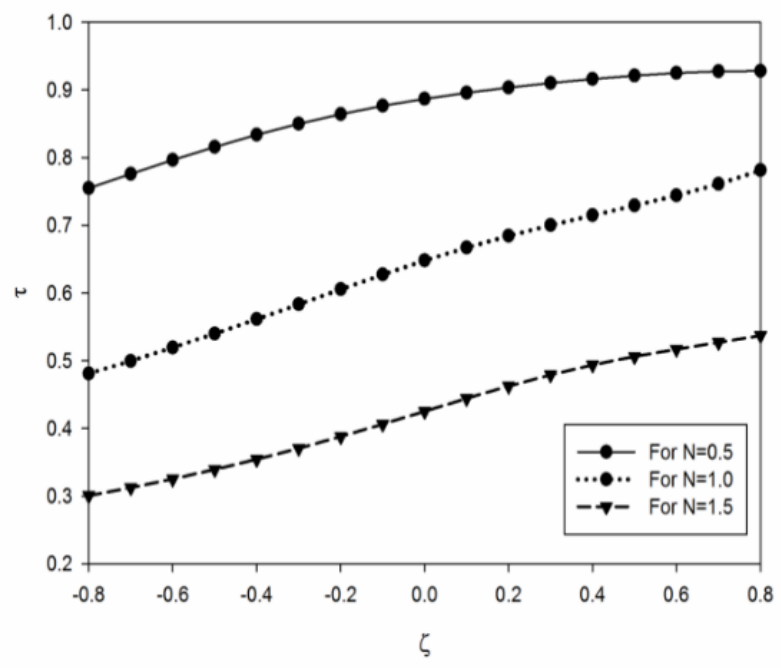

Figure 2. The variation of co-efficient $\tau$ for various values of $\mathrm{N}$ and $\zeta$

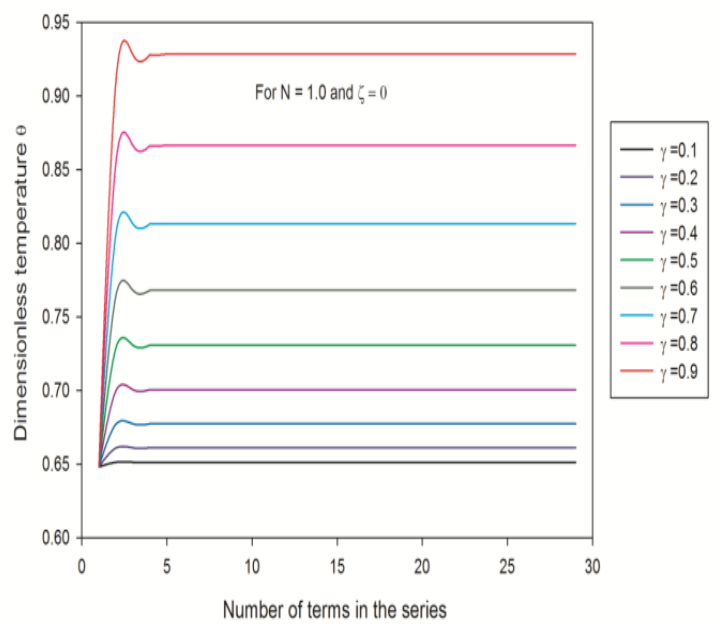

3(a)

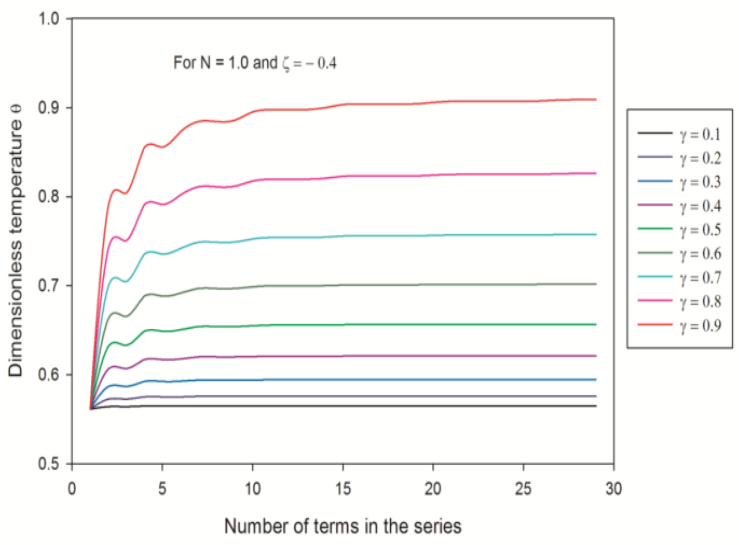

3(b)

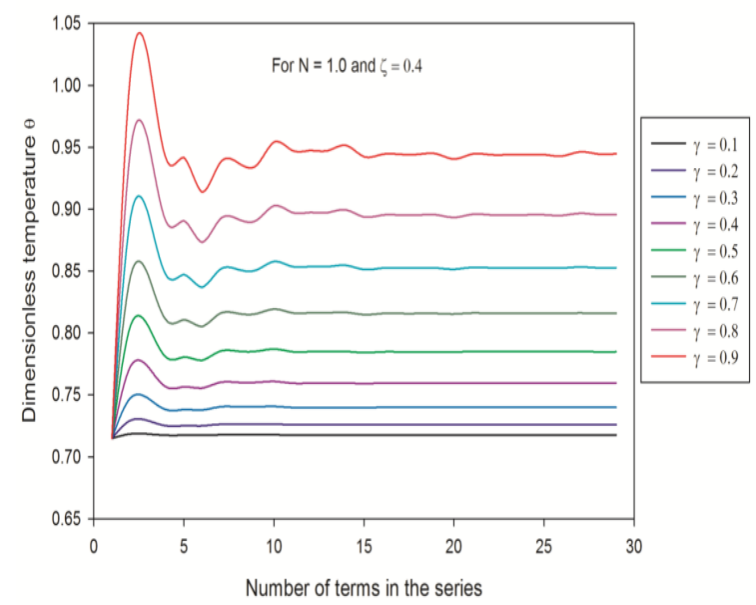

3(c)

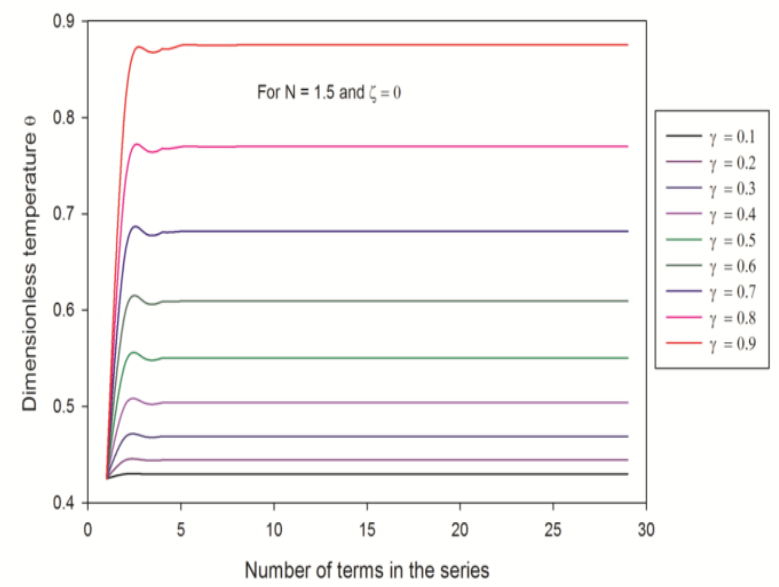

3(d)

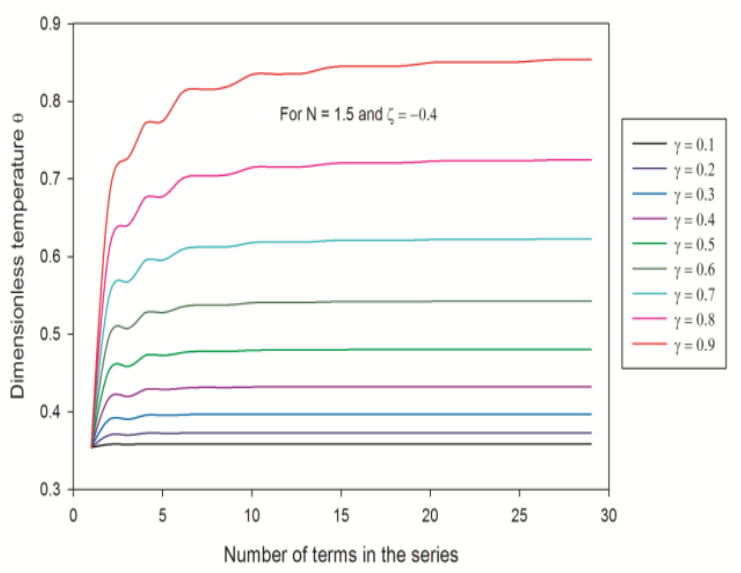

3(e) 


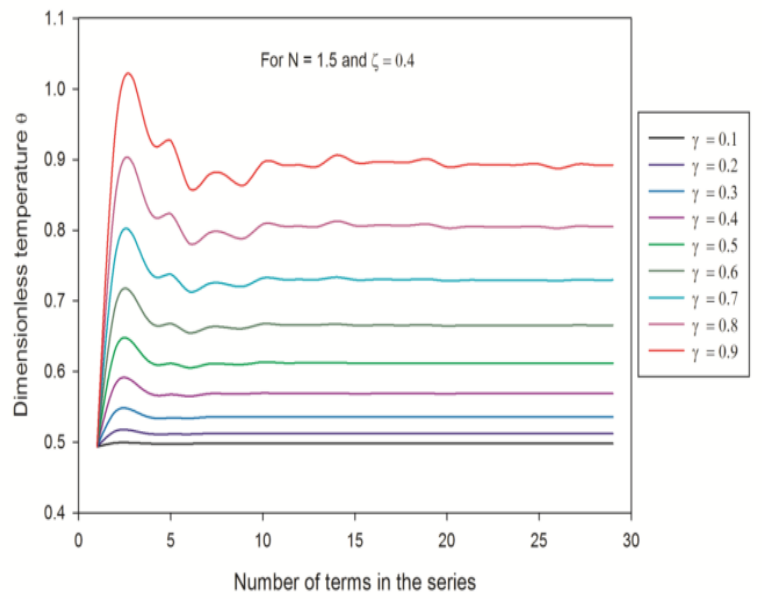

3(f)

Figure 3. Convergence chart based on no. of terms in the series for various fin parameters (a) $\mathrm{N}=1, \zeta=0$ (b) $\mathrm{N}=1, \zeta=$ 0.4 (c) $\mathrm{N}=1, \zeta=0.4$ (d) $\mathrm{N}=1.5, \zeta=0$ (e) $\mathrm{N}=1.5, \zeta=-0.4$ (f) $\mathrm{N}=1.5, \zeta=0.4$

The convergence of the solution for a given fin problem is greatly depends on the thermal conductivity parameter $\zeta$ compared with the convective-geometric property parameter $\mathrm{N}$ as depicted in figures 3 (a), (b) and (c). Equations (5) and (7) provided the information that the temperature distribution of the given fin problem is hardly influenced by the non-linear terms in that equations. The nonlinearities in the solutions of those equations are demonstrated in figures 3 (a), (b), (c), (d), (e) and (f) and it concludes the influence of the nonlinear terms over the solutions via various trends of convergence chart.

The number of terms required to obtain converged solution also depends on the thermal conductivity parameter as shown in figure sets 3 (b), (e) and 3 (c), (d) and the number of decompositions in nonlinear terms or the number of Adomian polynomials have to be improved for higher positive values of $\zeta$. Here, for given problem, boundary conditions and input parameter values, the solution obtained was better and it does not require more numbers of decomposition or Adomian polynomials.

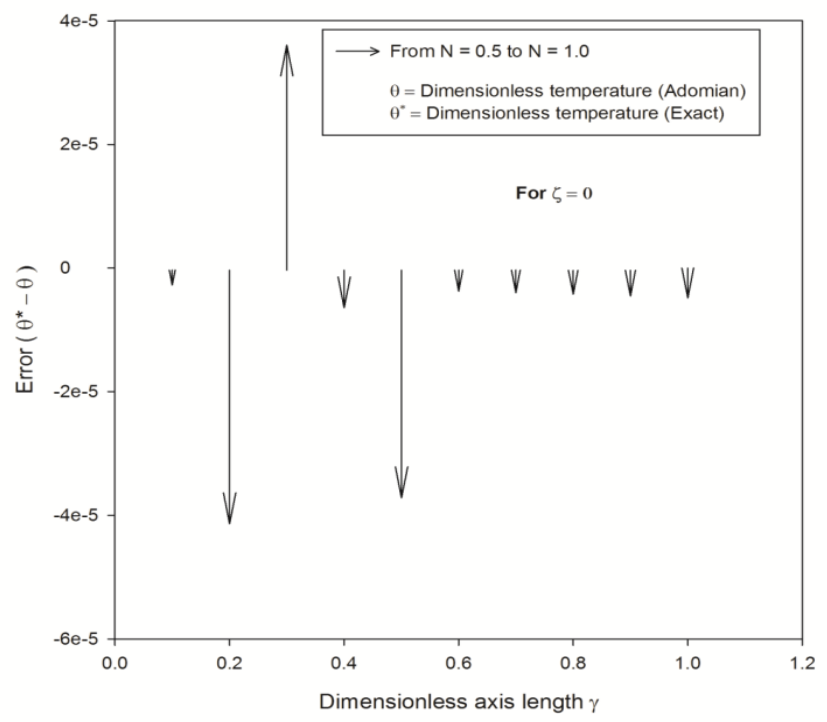

4(a)

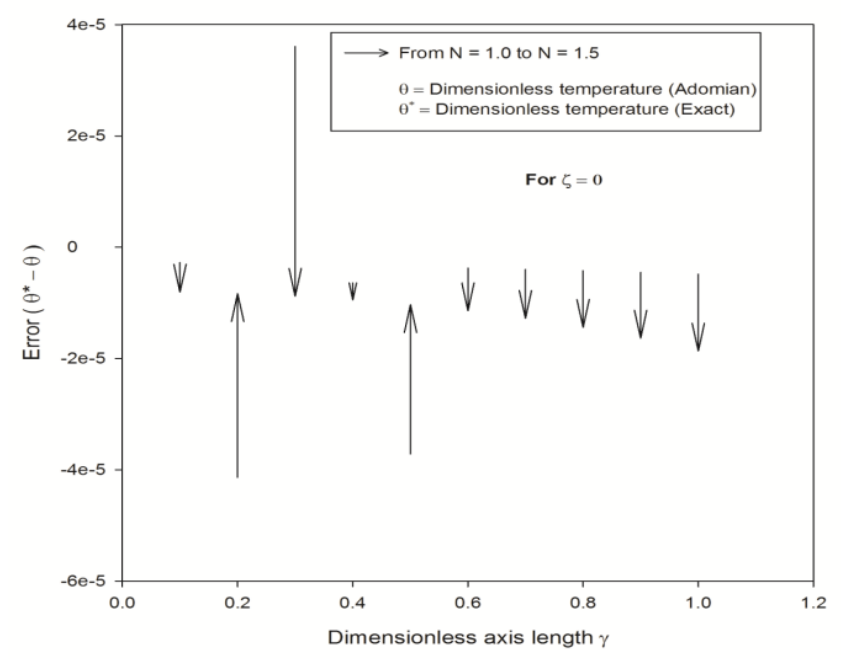

4(b)

Figure 4. Error in estimated values of dimensionless temperature of an isotropic fin at various axis lengths in a vector plot for different values of $\mathrm{N}$ (a) from $\mathrm{N}=0.5$ to $\mathrm{N}=$ 1.0 (b) from $\mathrm{N}=1.0$ to $\mathrm{N}=1.5$

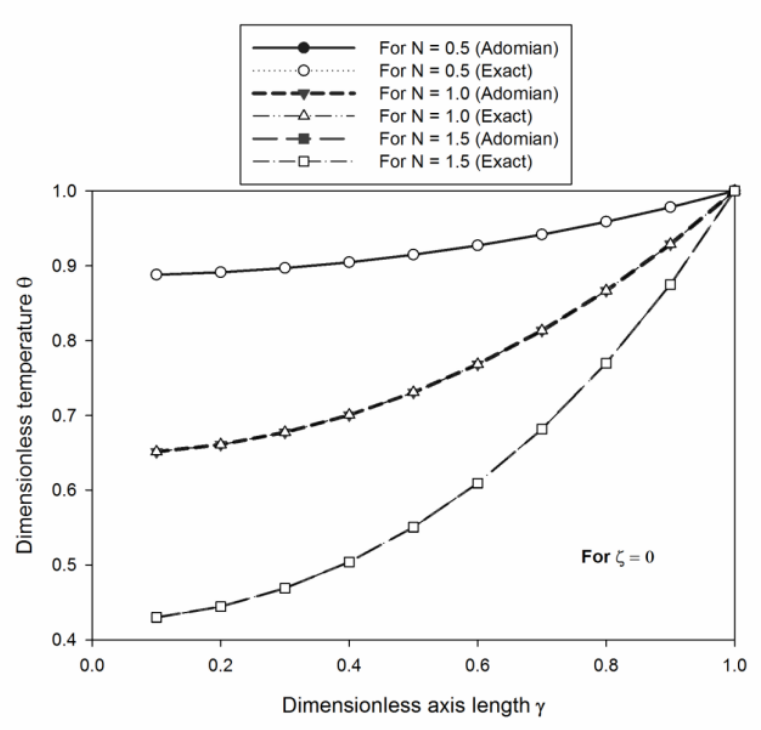

Figure 5. Comparison of present semi-analytical solutions with exact solutions of an isotropic fin for various value of geometric parameter $\mathrm{N}$

The exact solution of an isotropic fin was calculated based on the given boundary conditions and was used for error estimation of the present decomposition method based solutions. The error values of the estimated dimensionless temperature of the given problem are plotted in figure 4 (a) and (b). The vector plot represents the error values for an isotropic fin and exhibits the very less polluted prediction nature of the decomposition method.

For the given values of geometric-convective property parameter $\mathrm{N}$, the dimensionless temperature of an isotropic fin at various axis lengths based on both exact and decomposition methods was plotted in figure 5 and it shows the coincidence of the present solution with the exact solution. The plot shows the merit of decomposition method and the future scope of the method for an anisotropic material based heat conduction problems. 


\section{PERFORMANCE ANALYSIS AND OPTIMIZATION}

For performance analysis and optimization, the convectivegeometric property parameter $\mathrm{N}$ can be represented as a function of heat diffusion term Biot number and geometry design parameter $\psi$. The effect of design parameter over the heat transfer rate and efficiency of the fin can be analyzed and the optimum value of $\psi$ for maximum heat transfer rate is arrived by the method of conventional optimization for various Biot number

$\mathrm{N}^{2}=\frac{4 \mathrm{Bi}}{\psi^{2}}$

where

$\mathrm{Bi}=\frac{\mathrm{hLc}}{\mathrm{k}_{\mathrm{a}}}$ and $\psi=\frac{\mathrm{t}}{\mathrm{L}}$

The relationship between Biot number, convectivegeometric parameter and geometry design parameter was expressed in the following informative figure 6 .

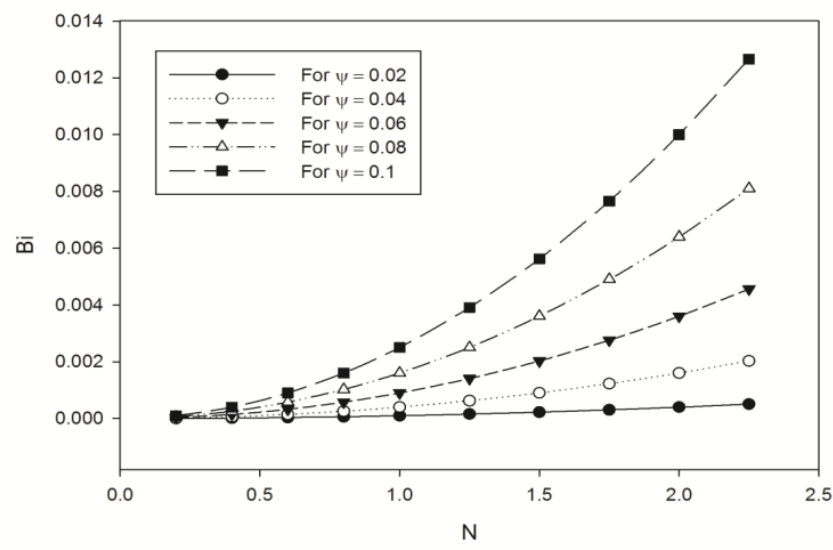

Figure 6. Relationship between N, Bi and $\psi$

The required dimensionless form of heat dissipation rate and efficiency of the fin is derived from the following conservative equations (30) and (31) and the steps are represented in the equations (32) and (33).

$\mathrm{Q}_{\mathrm{fin}}=\mathrm{L}\left(\mathrm{T}_{\mathrm{b}}-\mathrm{T}_{\mathrm{a}}\right) \int_{0}^{1} \operatorname{Ph} \theta(\gamma) \mathrm{d} \gamma$

$\eta_{\text {fin }}=\frac{Q_{\text {fin }}}{h_{A_{\text {fin }}\left(T_{b}-T_{a}\right)}}$.

The efficiency $\eta$ of given fin model [Appendix A] is represented with the help of dimensionless parameters as

$\eta=\int_{0}^{1} \theta(\gamma) \mathrm{d} \gamma$

Similarly, non-dimensional heat transfer rate per unit volume and unit width of fin is represented as

$Q_{n d}=(2 B i)^{4 / 3}\left(\sqrt{\frac{1}{\psi^{5}}}\right)^{2 / 3} \int_{0}^{1} \theta(\gamma) \mathrm{d} \gamma$

where Biot number and geometry design parameter are calculated based on equations (28) and (29).

The maximum heat dissipation rate occurs at optimum fin design characteristics and it can be achieved by searching optimum fin geometry design parameter based on heat diffusive parameter Biot number for the present problem. The optimization procedure is also executed to identify fin geometry design parameter $\psi$ where maximum heat dissipation occurs by expressing non-dimensional heat transfer rate as a function of $\mathrm{Bi}$ and $\psi$ or convective-geometry fin parameter N. For a given Biot number, the optimized value of fin geometry design parameter $\psi$ was calculated based on Newton-Raphson method. The optimization problem of both maximizing heat transfer rate and minimizing fin volume is formulated by Euler-Lagrange equation [15]. The dimensionless form of fin volume is represented as

$V=\frac{4 B i^{2}}{\psi}$

The dimensionless form of heat transfer rate at fin base as per Fourier's law of heat conduction is

$Q=\frac{Q_{\text {base }}}{k_{b}\left(T_{b}-T_{a}\right)}=\left.\psi \frac{\partial \theta}{\partial \gamma}\right|_{\gamma=1}$

The optimality criteria for present problem is constructed with Euler-Lagrange equation after eliminating Lagrange multipliers and represented as

$\left(\frac{\partial Q}{\partial B i}\right)\left(\frac{\partial V}{\partial \psi}\right)-\left(\frac{\partial Q}{\partial \psi}\right)\left(\frac{\partial V}{\partial B i}\right)=0$

From the equations (34), (35) \& (36), the functions for optimization problem set as

$S(B i, \psi)=\operatorname{Bi} \frac{\partial}{\partial B i}\left(\left.\frac{\partial \theta}{\partial \gamma}\right|_{\gamma=1}\right)+2 \psi \frac{\partial}{\partial \psi}\left(\left.\frac{\partial \theta}{\partial \gamma}\right|_{\gamma=1}\right)+$
$2\left(\left.\frac{\partial \theta}{\partial \gamma}\right|_{\gamma=1}\right)=0$

$R(B i, \psi)=\left\{\begin{array}{c}\psi\left(\left.\frac{\partial \theta}{\partial \gamma}\right|_{\gamma=1}\right)-Q=0 \\ \frac{4 B i^{2}}{\psi}-V=0\end{array}\right.$

The above non-linear equations are solved by NewtonRaphson method and the optimized values of $\mathrm{Bi}, \psi$ or $\mathrm{N}$ for given thermal conductivity parameter is calculated based on this iterative procedure.

$\left[\begin{array}{c}(B i)_{j+1} \\ (\psi)_{j+1}\end{array}\right]=\left[\begin{array}{c}(B i)_{j} \\ (\psi)_{j}\end{array}\right]-\left[\begin{array}{ll}\left(\frac{\partial S}{\partial B i}\right)_{j} & \left(\frac{\partial S}{\partial \psi}\right)_{j} \\ \left(\frac{\partial R}{\partial B i}\right)_{j} & \left(\frac{\partial R}{\partial \psi}\right)_{j}\end{array}\right]^{-1}\left[\begin{array}{l}S\left(B i_{j}, \psi_{j}\right) \\ R\left(B i_{j}, \psi_{j}\right)\end{array}\right]$

The converged results of the above iterative problem were demonstrated in following section and the effect of thermal conductivity parameter on optimized fin convective-geometry parameters has been discussed elaborately in the following sections.

\section{RESULTS AND DISCUSSION}

The following sets of plot in figure 7 designate the importance of thermal conductivity parameter in fin designing 
of temperature dependent soft materials. The dimensionless temperature profile of the temperature dependent thermal conductivity fin follows the diminishing nature for the increasing nature of the convective-geometric property parameter $\mathrm{N}$ as stated in figure 7 . Lower value of $\mathrm{N}$ describes the high convective nature and smaller design parameters of the fin and it shows good effectiveness compared with higher values of $\mathrm{N}$. The dimensionless temperature distribution over the longitudinal axis of the fin is plotted in the figures 7(a), 7(b) and 7(c). At fin base $\theta$ value is maximized and it decreases in the direction of fin tip due to the effect of conductive resistance of the fin material. Due to the rise in $\mathrm{N}$ value, the dimensionless temperature at different axis length along the longitudinal axis direction falls and follows the reducing nature as displayed in figure 7 .

The major effect of thermal conductivity parameter $\zeta$ was elaborated in figure 7(a) for lower value of $\mathrm{N}$ (substantially a shorter fin). The negative slope of thermal conductivity value $\zeta$ has greater effect on the performance of fins for smaller value of $\mathrm{N}$. The significant amount of drop in temperature has occurred as a result of decreasing nature of negative slope of thermal conductivity $\zeta$ and it has been clearly displayed in figure 7(a). In the same figure 7(a), the effect of positive slope value of thermal conductivity $\zeta$ has been addressed and strongly stated that the positive slope value of thermal conductivity does not supplements the considerable amount of performance improvement to the fin system.

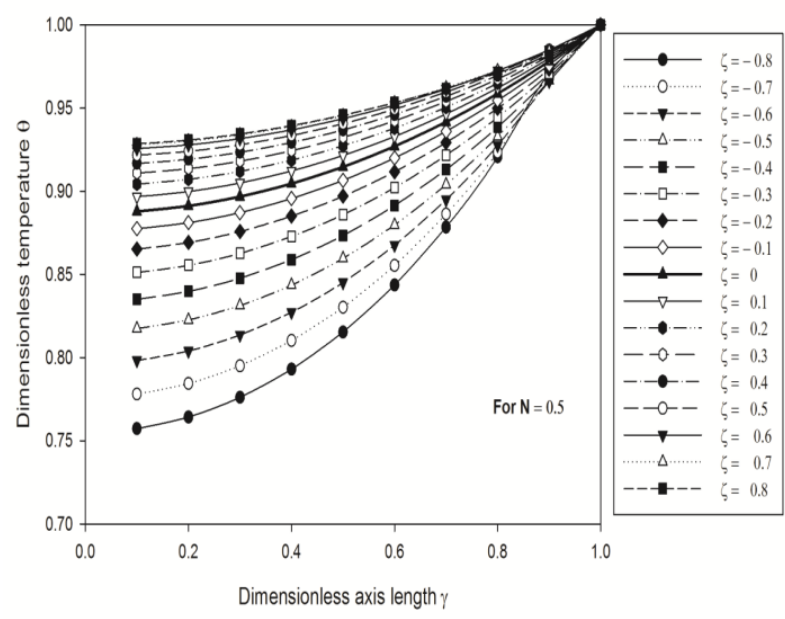

7(a)

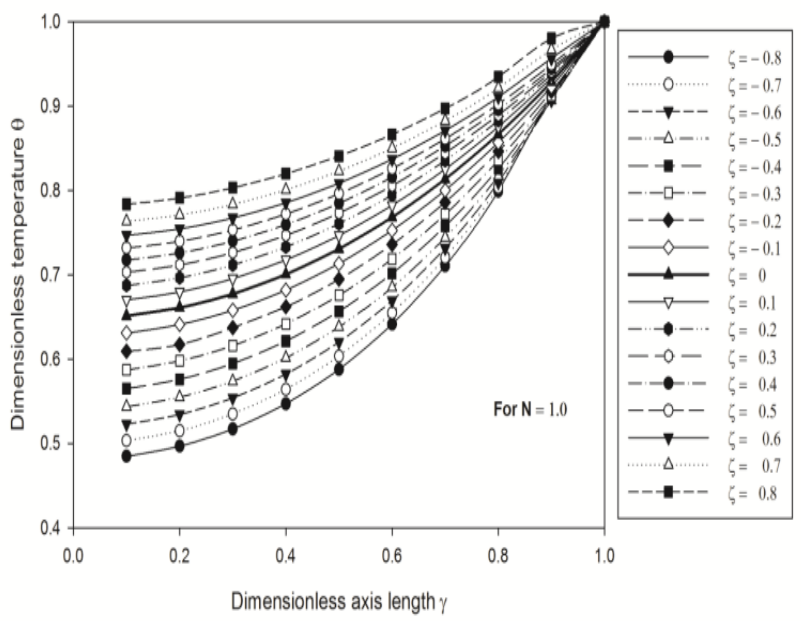

7(b)

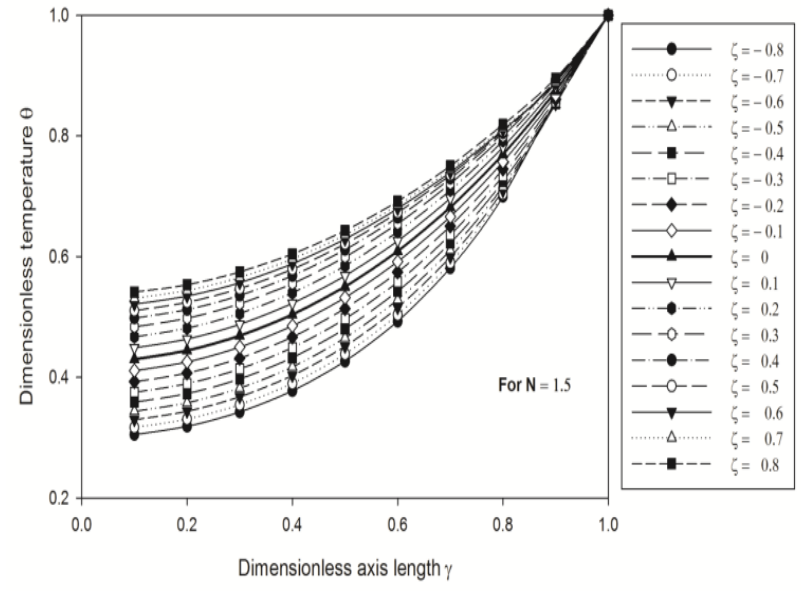

$7(\mathbf{c})$

Figure 7. Dimensionless temperature distribution in axial direction of the fin based on different $\zeta$ values (a) For $\mathrm{N}=0.5$

(b) For $\mathrm{N}=1.0$ and (c) For $\mathrm{N}=1.5$.

For higher values of $\mathrm{N}$, the effect of thermal conductivity parameter $\zeta$ on the fin performance is not much significant compared with lower value of $\mathrm{N}$ as shown in figure 7(b) and 7(c). The longer fins are exhibiting very minor changes in its performance for various values of thermal conductivity parameter $\zeta$. The longer fins can accommodate high heat flux on its volume and the average temperature of the system is considerably lower than shorter fins. The effect of temperature dependent thermal conductivity on fin performance can be tackled with the help of longer fins with high convective environment but the longer fins have less effectiveness which affects the heat transfer performance of the system. So, for heat transfer enhancement problems, shorter fins can be accommodated with high convective environment to meet the variable thermal conductivity nature of the fins.

For a fixed value of thermal conductivity parameter $\zeta$ with given specified perimeter of a fin, the dimensionless heat transfer rate per unit volume increases and then marginally decreases with respect to an increase in $\mathrm{N}$ value as shown in figure 8. After optimum fin length $\mathrm{L}$, heat transfer rate decreases due to hike in overall heat transfer resistance. The optimum fin length value gradually increases for higher values of thermal conductivity parameter $\zeta$.

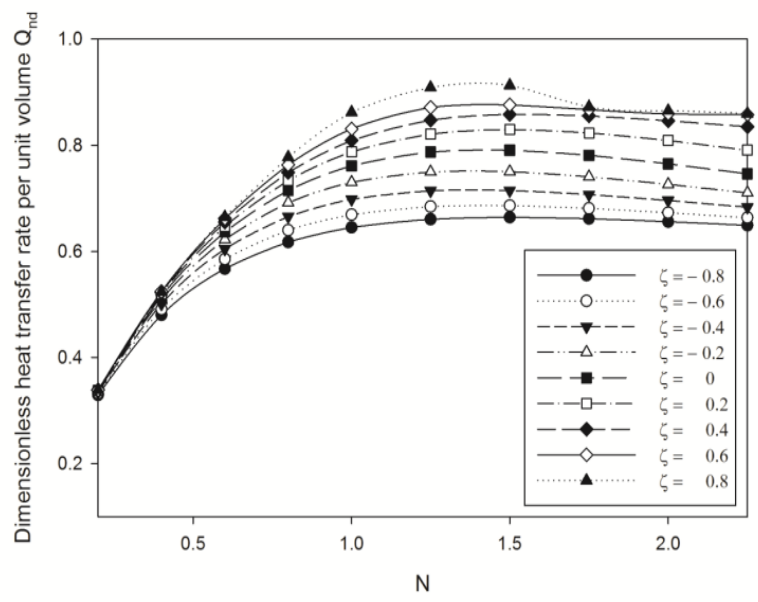

Figure 8. Dimensionless heat transfer rate per unit volume for various $\mathrm{N}$ and $\zeta$ values. 


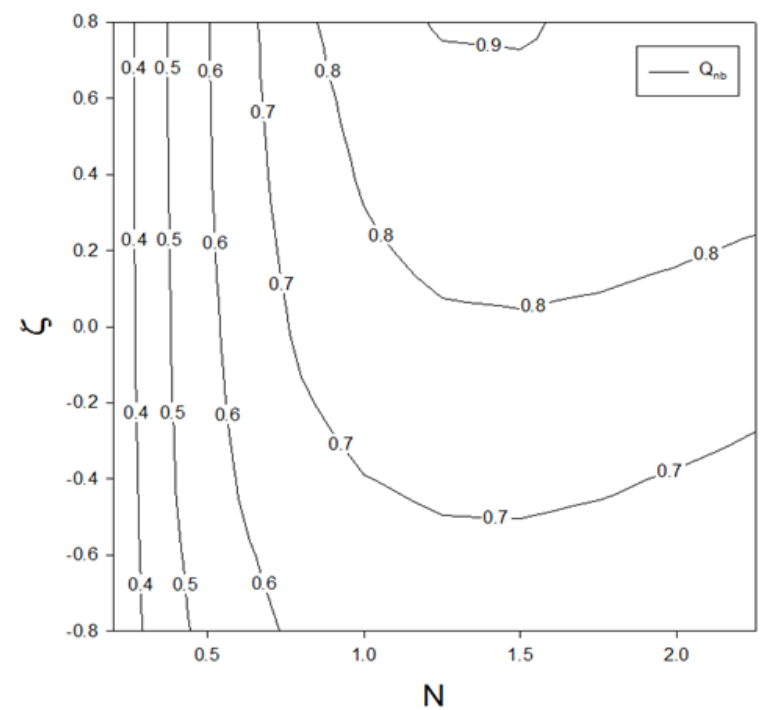

Figure 9. Contour plot of heat transfer rate for various $\mathrm{N}$ and $\zeta$ values

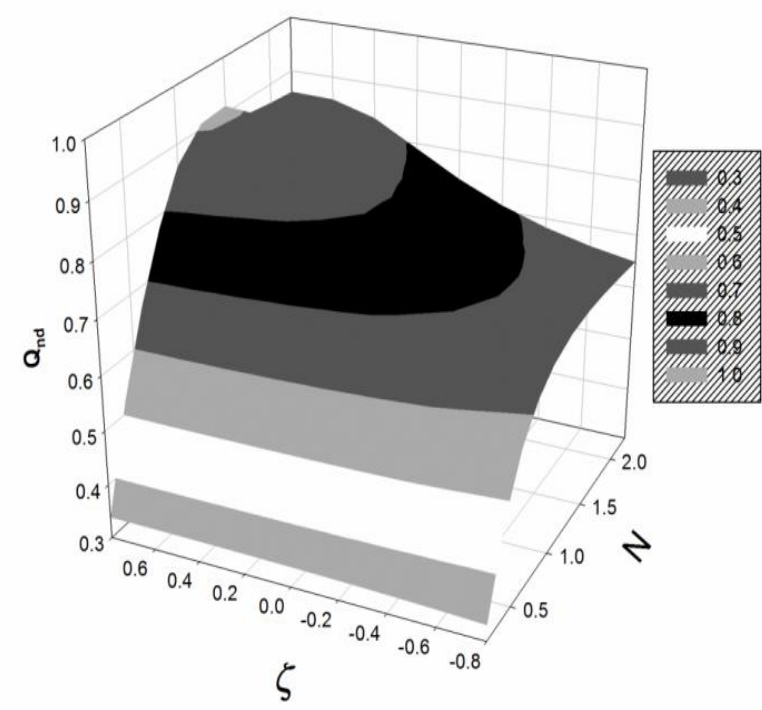

Figure 10. 3D surface plot of heat transfer rate for various $\mathrm{N}$ and $\zeta$ values

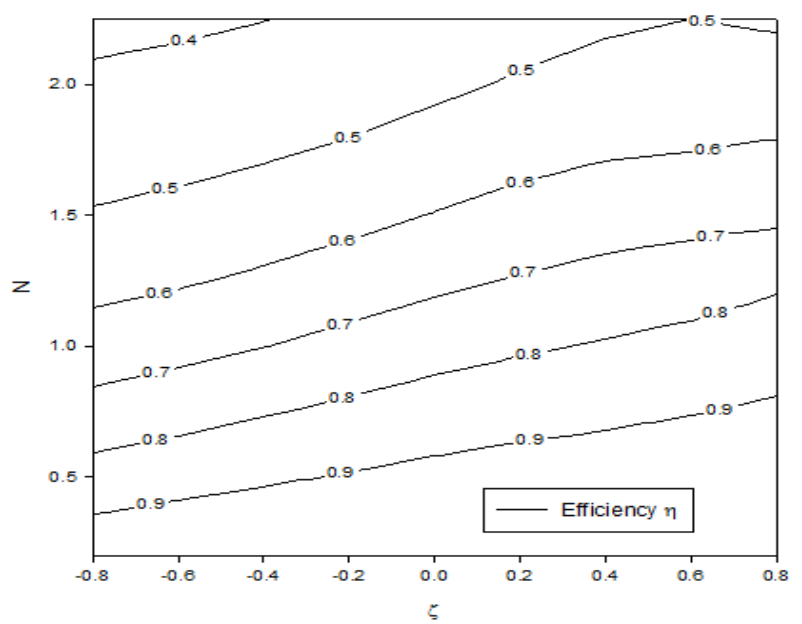

Figure 11. Efficiency contour plot
The effect of thermal conductivity parameter $\zeta$ and convective-geometric parameter $\mathrm{N}$ on overall heat transfer rate of the fin system has been clearly demonstrated in the following figures 9 and 10 as 2D and 3D contour plots.

The figure 11 depicts the efficiency of the fin system based on independent fin parameters $\mathrm{N}$ and $\zeta$. The efficiency of fin increases as the value of thermal conductivity parameter $\zeta$ increases and then it decreases as the value of $\mathrm{N}$ increases. The shorter fin with lower $\mathrm{N}$ value has greater efficiency compared with longer fins for a fixed value of negative slope of thermal conductivity parameter $\zeta$. And also the gradient field of the efficiency of the fin shows the direction of maximum performance as the location of lower $\mathrm{N}$ value with higher $\zeta$ value.

The higher values of geometry design parameter $\psi$ (considerably very thin fin with shorter length in nature), the required optimum Biot number was significantly fluctuated for negative directional values of $\zeta$ and has no effect for larger positive values of $\zeta$ as shown in figure 12 .

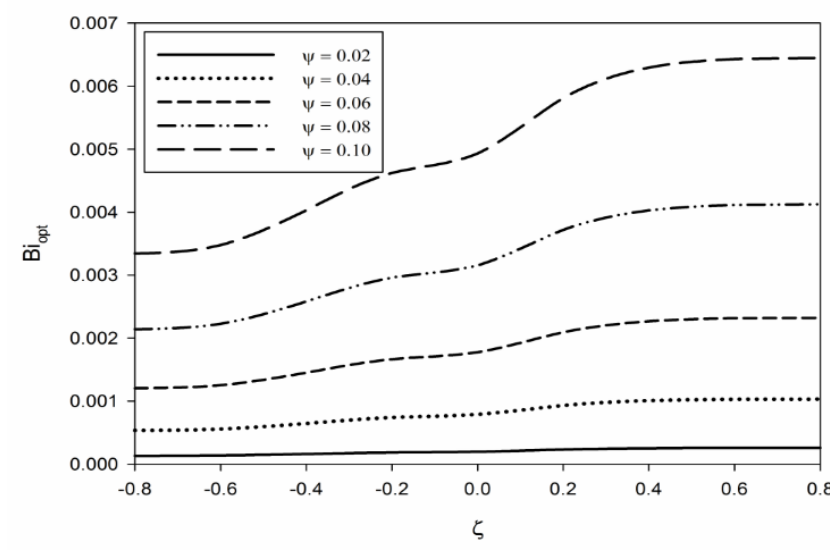

Figure 12. Effect of thermal conductivity parameter on optimum Biot number of the fin for regulated fin geometry design parameter

Similarly, a smaller value of geometry design parameter, the value of $\zeta$ has no effect of optimized Biot number values. For a fixed convective environment, the required optimum fin geometry design parameter $\psi$ considerably increases for negative directional flow of slope value of thermal conductivity $\zeta$ as stated in plot 13 .

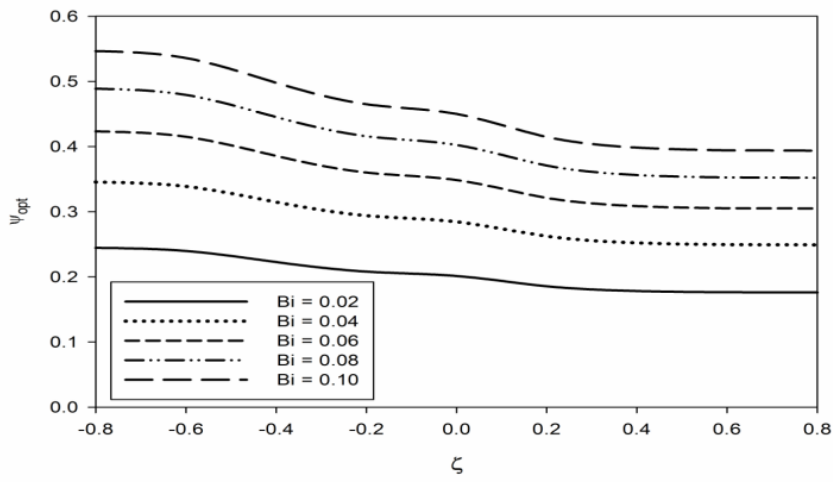

Figure 13. Effect of thermal conductivity parameter on optimum fin geometry design parameter for various heat transfer zones 
From the figure 13, the requirement of fin geometry modification for negative value of $\zeta$ has been clearly depicted and for a fixed convective environment, the further improvement of geometry design parameter for larger values of $\zeta$ does not add any value to the system performance.

\section{CONCLUSION}

The Adomian Decomposition Method (ADM) was used to analyze the heat transfer performance of a nonlinear, convective, straight, rectangular fin with temperature dependent thermal conductivity property. This ADM method provides an approximate exact solution of the nonlinear equations without any linearization scheme. The results obtained from this method have a nature of fast convergence, high accuracy and significantly in explicit form.

From the present analytical investigation, the following ultimate interpretations can be pinched:

1. The temperature distribution in the temperature dependent thermal conductivity fin greatly depends on the parameters $\mathrm{N}$ and $\zeta$. The negative value of $\zeta$ has high impact on the temperature distribution in the fin compared with positive values of $\zeta$. Similarly, the lower value of $\mathrm{N}$ leads to very high temperature distribution in the fin along the longitudinal axis direction compared with higher values of $\mathrm{N}$.

2. For a fixed $\mathrm{N}$ value, the dimensionless heat transfer rate increases rapidly with the increasing nature of $\zeta$. Similarly, for a fixed value of $\zeta$, heat transfer rate of the fin increases up to optimum $\mathrm{N}$ value and then decreases for further enlargement in $\mathrm{N}$ value.

3. For a fixed perimeter of the fin, the optimum length of the fin increases with respect to positive flow nature of the thermal conductivity parameter $\zeta$.

4. The shorter fin with higher thermal conductivity parameter $\zeta$ has very high fin efficiency and for a negative value of $\zeta$, optimum fin length is minimum compared with positive values of it.

5. Finally, the present optimized values of fin geometry design parameter $\psi$ may help to design a fin with above mentioned nature and boundary conditions.

\section{REFERENCES}

[1] Incropera FP, Dewitt DP. (1996). Introduction to Heat Transfer. Third ed., Wiley, New York.

[2] Kraus AD. (1972). Extended Surface Heat Transfer. McGraw-Hill, New York.

[3] Grimvall G. (1999). Thermophysical properties of materials. Elsevier.

[4] Choi S, Kim J. (2013). Thermal conductivity of epoxy composites with a binary-particle system of aluminum oxide and aluminum nitride fillers. Compos. Part B Eng. 51:

$140-147$ https://doi.org/10.1016/j.compositesb.2013.03.002

[5] Bigg D, Stickford G, Talbert S. (1989). Applications of polymeric materials for condensing heat exchangers. Polymeric Engineering and Science 29: 1111-1116. https://doi.org/10.1002/pen.760291607

[6] Vadivelu MA, Ramesh Kumar C, Girish MJ. (2016). Polymer composites for thermal management: A review. Composite Interfaces http://dx.doi.org/10.1080/09276440.2016.1176853

[7] Hung HM, Appl FC. (1967). Heat transfer of thin fins with temperature dependent thermal properties and internal heat generation. ASME J Heat Transfer 89: 155161. https://dx.doi.org/10.1115/1.3614342

[8] Unal HC. (1986). Determination of the temperature distribution in an extended surface with a non-uniform heat transfer coefficient. Int J Heat Mass Transfer 28: 2279-84. https://doi.org/10.1016/0017-9310(85)90046-8

[9] Meyer GE. (1971). Analytical Methods in Heat Conduction, McGraw-Hill, New York.

[10] Muzzio A. (1976). Approximate solution for convective fins with variable thermal conductivity. J Heat Transfer Trans. ASME 98: 680-682. https://doi.org/10.1115/1.3450623

[11] Thongmoon M, Pusjuso S. (2010). The numerical solutions of differential transform method and Laplace method for a system of differential equations. Nonlinear Analysis: Hybrid Systems 4: 425-431. https://doi.org/10.1016/j.nahs.2009.10.006

[12] Ozkan O. (2010). Numerical implementation of differential transformations method for integrodifferential equations. International Journal of Computer Mathematics $87:$ 2786-2797. https://doi.org/10.1080/00207160902795627

[13] Bert CW. (2002). Application of differential transform method to heat conduction in tapered fins. ASME Journal of Heat Transfer 124: 208-209. https://doi.org/10.1115/1.1423316

[14] Chu HP, Lo CY. (2008). Application of the hybrid differential transform-finite difference method to nonlinear transient heat conduction problem. Numerical Heat Transfer Part A 53: 295-307. Https://dx.doi.org/10.1080/10407780701557931

[15] Jang MJ, Chen CL, Liu YC. (2001). Two-dimensional differential transform for partial differential equations. Appl. Math. Comput. 121: 261-270. https://doi.org/10.1016/S0096-3003(99)00293-3

[16] Adomian G. (1988). A review of the decomposition method in applied mathematics. J. Math. Anal. Applications 135(2): 501-544. https://doi.org/10.1016/0022-247X(88)90170-9

[17] Adomian G. (1994). Solving Frontier Problems in Physics: The Decomposition Method. Kluwer Academic Publisher, Dordrecht

[18] Adomian G. (1985). On the solution of algebraic by the decomposition method. J. Math. Anal. Applications 105(1): 141-166. https://doi.org/10.1016/0022247X(85)90102-7

[19] Adomian G. (1988). Nonlinear stochastic system theory and application to physics. Kluwer Academic Publisher, Dordrecht.

[20] Adomian G. (1991). Solving frontier problems modeled by nonlinear partial differential equations. Comput. Math. Appl. 22(8): 91-94. https://doi.org/10.1016/08981221(91)90017-X

[21] Shih TM. (1984). In: Numerical Heat Transfer. Springer, New York.

\section{NOMENCLATURE}

$A_{c}$ fin cross sectional area in $\mathrm{m}^{2}$

$\mathrm{Bi}$ Biot number 
$\mathrm{h}$ convective heat transfer co-efficient in $\mathrm{W} / \mathrm{m}^{2}-\mathrm{K}$

$\mathrm{k}$ thermal conductivity in $\mathrm{W} / \mathrm{m}-\mathrm{K}$

$\mathrm{L}$ length of the fin in $\mathrm{m}$

$\mathrm{N}$ convective-geometry parameter / fin parameter

$\mathrm{P}$ perimeter of the fin in $\mathrm{m}$

$\mathrm{Q}$ dimensionless heat transfer rate

$\mathrm{T}$ temperature in $\mathrm{K}$

$\mathrm{t}$ fin thickness in $\mathrm{m}$

$\mathrm{V}$ dimensionless fin volume

$\mathrm{x}$ axial direction

\section{Greek symbols}

$\Omega$ ratio of slope of thermal conductivity - temperature plot and thermal conductivity $\mathrm{k}_{\mathrm{a}}$

$\theta$ dimensionless temperature distribution

$\gamma$ dimensionless axial length

$\zeta / \zeta$ thermal conductivity parameter

$\sigma$ temporary function

$\lambda$ independent variable of temporary function

$\tau$ co-efficient value formed by constant terms of the solution due to given boundary condition

$\psi$ fin thickness to length ratio

$\eta$ fin efficiency

\section{Subscripts}

a ambient

b base c characteristic

nd non-dimensional

\section{APPENDIX A}

The efficiency of the fin $\eta$ as a function of independent variables $\mathrm{N}$ and $\zeta$

$$
\begin{aligned}
\eta=\tau+\frac{1}{6} \tau N^{2}+\frac{1}{120} & \tau N^{4}-\frac{1}{6} \zeta \tau^{2} N^{2}+\frac{1}{5040} \tau N^{6}-\frac{1}{24} \zeta \tau^{2} N^{4} \\
& +\frac{1}{6} \zeta^{2} \tau^{3} N^{2}+\frac{1}{362880} \tau N^{8}-\frac{1}{315} \zeta \tau^{2} N^{6} \\
& +\frac{1}{10} \zeta^{2} \tau^{3} N^{4}-\frac{1}{6} \zeta^{3} \tau^{4} N^{2}+\frac{1}{39916800} \tau N^{10} \\
& -\frac{37}{181440} \zeta \tau^{2} N^{8}+\frac{7}{360} \zeta^{2} \tau^{3} N^{6}-\frac{1}{6} \zeta^{3} \tau^{4} N^{4} \\
& +\frac{1}{6} \zeta^{4} \tau^{5} N^{2}+\frac{1}{6227020800} \tau N^{12} \\
& -\frac{19}{3326400} \zeta \tau^{2} N^{10}+\frac{407}{181440} \zeta^{2} \tau^{3} N^{8} \\
& -\frac{5}{72} \zeta^{3} \tau^{4} N^{6}+\frac{31}{120} \zeta^{4} \tau^{5} N^{4}-\frac{1}{6} \zeta^{5} \tau^{6} N^{2} \\
& +\frac{1}{128314368000} \tau N^{14} \\
& -\frac{1.943144305}{10000000} \zeta \tau^{2} N^{12} \\
& +\frac{2.205337101}{10000} \zeta^{2} \tau^{3} N^{10}-\frac{677}{40320} \zeta^{3} \tau^{4} N^{8} \\
& +\frac{317}{1680} \zeta^{4} \tau^{5} N^{6}-\frac{47}{120} \zeta^{5} \tau^{6} N^{4}+\frac{1}{6} N^{2}+
\end{aligned}
$$

